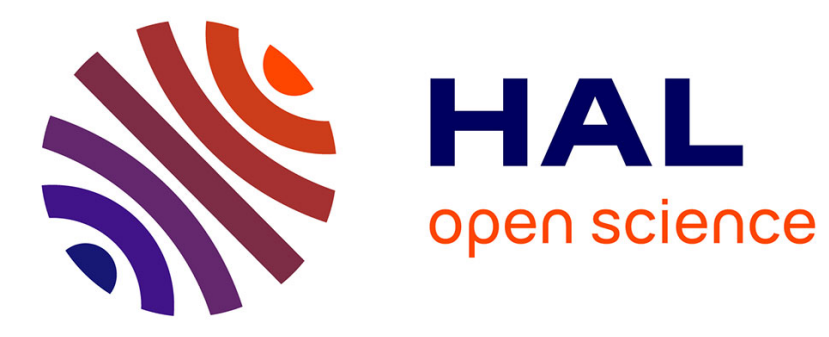

\title{
Energy-efficient discovery process for mMTC applications
}

Cesar Vargas Anamuro, Nadège Varsier, Jean Schwoerer, Xavier Lagrange

\section{To cite this version:}

Cesar Vargas Anamuro, Nadège Varsier, Jean Schwoerer, Xavier Lagrange. Energy-efficient discovery process for mMTC applications. WNMC 2019: 12th IFIP Wireless and Mobile Networking Conference, Sep 2019, Paris, France. 10.23919/WMNC.2019.8881827 . hal-02100252

\section{HAL Id: hal-02100252 \\ https://hal.science/hal-02100252}

Submitted on 15 Apr 2019

HAL is a multi-disciplinary open access archive for the deposit and dissemination of scientific research documents, whether they are published or not. The documents may come from teaching and research institutions in France or abroad, or from public or private research centers.
L'archive ouverte pluridisciplinaire HAL, est destinée au dépôt et à la diffusion de documents scientifiques de niveau recherche, publiés ou non, émanant des établissements d'enseignement et de recherche français ou étrangers, des laboratoires publics ou privés. 


\title{
Energy-efficient discovery process for mMTC applications
}

\author{
Cesar Vargas Anamuro*†, Nadège Varsier*, Jean Schwoerer* and Xavier Lagrange ${ }^{\dagger}$ \\ * Orange Labs, Meylan, France \\ Email: \{cesaraugusto.vargasanamuro, nadege.varsier, jean.schwoerer\}@orange.com \\ ${ }^{\dagger}$ IMT Atlantique/IRISA, Rennes, France \\ Email: xavier.lagrange@imt-atlantique.fr
}

\begin{abstract}
Reducing energy consumption is fundamental to address the requirements of Massive Machine-Type Communication (mMTC) applications. In this sense, D2D relaying could play an important role in some scenarios. D2D discovery process is a key phase of D2D relaying since in this phase the MTC device (MTD) has to select an optimal relay. This process should ensure low energy consumption and low complexity while taking into account the specific constraints of the MTDs.

In this paper, we analyze the total (i.e., during the discovery phase and the data transmission phase) energy consumed by the MTD when it uses a D2D relaying mechanism. We propose a simple distributed discovery protocol, which allows reducing the MTD energy consumption. Our numerical and simulation results confirm that when using the proposed discovery mechanism, the total MTD energy consumption can be significantly reduced depending on the data packet size and the location of the MTD with respect to the base station.
\end{abstract}

Index Terms-Energy-efficient, mMTC, D2D discovery, relay selection

\section{INTRODUCTION}

The future Fifth Generation (5G) of cellular networks aims to support three major service groups: Enhanced Mobile BroadBand (eMBB), Ultra-Reliable Low Latency Communications (URLLC), and Massive Machine Type Communications (mMTC) [1] [2]. Each of these services has different characteristics and requirements. eMBB can be seen as an extension of the $4 \mathrm{G}$ networks, which focuses mainly on the improvement of the data rate. URLLC groups the applications that need high level of reliability and low latency while mMTC services are characterized by a large number of Machine-type Devices (MTDs). The MTDs are usually low-complexity devices that sporadically transmit small data packets. Moreover, most of these devices are usually equipped with low-capacity batteries, which must keep the MTD operational for many years. Minimizing energy consumption due to the transmission of data has a positive impact on the battery lifetime of the MTDs.

The use of Device-to-Device (D2D) communications to reduce the MTD energy consumption has been proposed in the literature [3] [4] [5]. An MTD in poor coverage conditions sends its data to a nearby User Equipment (UE), which acts as a relay. The main advantage of such configuration is a better link performance since the relay can be closer to the MTD than the Base Station (BS). However, the MTD must search for a relay before transmitting its data. This phase, called the discovery phase, causes additional energy consumption, which is not negligible for small data transmissions.

The use of relays to improve the energy efficiency has been well studied [6] [7] [8]. Authors in [6] analyze the total energy consumption (including the energy cost of acquiring channel information) based on a general relay selection approach. In [7] the authors propose a relay selection scheme based on 802.11 RTC/CTS (Request-to-Send/Clear-to-Send) protocol, where an optimal relay is selected in order to maximize the energy efficiency. In [8], the relay selection strategy is analyzed considering the double auction theory. However, in all these works, the total energy consumption is computed taking into account also the energy consumed by the relay. This is a reasonable approach in networks where all devices have the same characteristics (e.g., wireless sensor networks).

In this work, we consider two groups of devices with different characteristics, the MTDs (energy-constrained devices) and the UEs (non-energy constrained devices) as in [5]. Our objective is to analyze the energy consumed by an MTD when it uses a UE as a relay to transmit its data. From this analysis, we determine in which cases it is convenient for the MTD to use a D2D relay mechanism instead of a direct transmission to the BS.

A D2D relay mechanism is composed of two phases: the discovery phase, and the data transmission phase. If no relay is selected at the end of the discovery phase, the MTD transmits data to the BS, which may induce a large energy consumption. If a relay is selected, its location is very important because the closer the relay is to the MTD, the lower the MTD energy consumption will be during the data transmission phase. Therefore, the discovery phase is the key phase to minimize the total MTD energy consumption (i.e., the energy consumed in both phases).

In this paper we propose a discovery and relay selection process in a distributed manner (without the participation of the BS) inspired by the RTS/CTS protocol as in [7] [9] [10]. This protocol is suitable for mMTC applications due to its simplicity and limited signal overhead. In [9] and [10], the neighbor UE with the best channel condition UE-BS is selected as a relay. While [9] shows simulation and analytical results, the results were obtained only through simulations in [10]. In [7] the authors take the power control into account. Our protocol differs from [7] [9] [10] as follows: (i) it selects 
a non-energy constrained device (UE) as relay; (ii) it selects a relay close to the MTD by limiting the discovery area; (iii) it solves the hidden node problem thanks to a feedback packet; (iv) and finally it considers dynamic data rate adaptation.

First, we analyze the relay discovery probability and the average number of slots used in the contention process. From these parameters, we derive the energy consumption in the discovery phase and in the data transmission phase. In order to minimize the MTD energy consumption, we derive the optimal contention window size and the optimal radius of the discovery area. Finally, we compare the energy consumption in cellular mode and the energy consumption in D2D mode taking into account the optimal values.

The remainder of this paper is organized as follows. Section II presents the system model. The relay discovery probability is derived in section III. In Section IV, we analyze the number of slots used in the contention process. We compare the energy consumption in cellular and D2D modes in Section V while in Section VI we present the numerical results. Finally, conclusions and future works are given in Section VII.

\section{System Model}

\section{A. Network Model}

We consider a single cell in which UEs and MTDs are randomly distributed. The locations of UEs form a homogeneous Poisson point process (PPP) $\Phi_{u}$ with density $\lambda_{u}$ in $\mathbb{R}^{2}$. All the devices (MTDs and UEs) are in down-link coverage. MTDs with an unfavorable link budget have up-link coverage at the cost of higher energy consumption. In order to reduce its energy consumption, the MTD may use a nearby UE as a relay. This approach consists of two phases:

1) Discovery phase: The MTD searches for a nearby UE, which can serve as a relay.

2) Data transmission phase: If the MTD finds a relay, it transmits its data to the selected relay using a D2D communication, then the selected relay transmits the MTD data to the BS using a cellular communication. Otherwise, the MTD transmits its data directly to the BS using a cellular communication.

In our analysis, we do not consider the energy consumed by the relay. We assume that the BS allocates dedicated resources for D2D links (i.e., there is no interference between D2D links and cellular links). In order to keep the analysis tractable, we make the following assumptions:

- The path loss is distance-based (i.e., without considering fading and shadowing effects).

- Devices (MTDs and UEs) transmit at a fixed transmission power and have a bandwidth of $B_{w} \mathrm{~Hz}$.

- The channel is reciprocal (i.e., the channel from point A to $\mathrm{B}$ is the same as the channel from B to A).

\section{B. Propagation model}

There are two communication modes: cellular mode and D2D mode. In cellular mode, the MTD communicates directly with the BS while in D2D mode the MTD communicates with

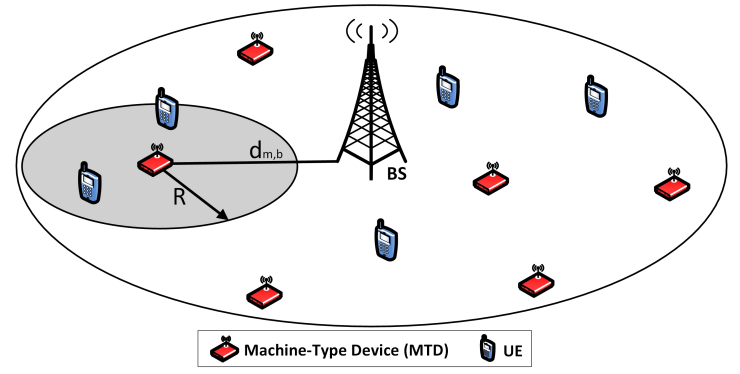

Fig. 1. Network model.

the relay (UE). We consider the path loss model described in [11] which is based on the micro-urban channel models from ITU [12].

We consider only non-line-of-sight (NLoS) scenarios. Thus the path loss models for carrier frequency $\left(f_{c}\right) 900 \mathrm{MHz}$ are given in Table I, where $d_{m, b}$ and $d_{m, u}$ are the MTD-BS and the MTD-UE distances (in meters), respectively.

TABLE I

PATH LOSS MODELS FOR NLOS WITH DISTANCE IN METERS, $f_{c}=900$ $\mathrm{MHz}$

\begin{tabular}{|c|c|}
\hline Communication mode & Path loss $(\mathbf{d B})$ \\
\hline Cellular & $L_{m, b}=21.53+36.7 \log _{10}\left(d_{m, b}\right)$ \\
\hline D2D & $L_{m, u}=17.62+40 \log _{10}\left(d_{m, u}\right)$ \\
\hline
\end{tabular}

\section{Discovery protocol description}

The proposed protocol uses a distributed relay selection approach (i.e., the BS does not participate in the relay selection process). When the MTD wakes up to transmit its data, it first waits for the synchronization messages sent by the BS. Then, the procedure consists of the following steps:

1) The MTD measures the path loss value $L_{m, b}$ between itself and the BS when it reads the network configuration messages sent by the BS. Based on this value, it defines the contention window size $W$ and the path loss threshold between itself and a nearby UE $\left(L_{t h}\right)$. Then it broadcasts a Request-for-Relay (RR) packet that carries the values of $W$ and $L_{t h}$ and waits for a response from a UE (contention process). The maximum waiting time is $W$ time slots.

2) A UE that receives an RR packet measures the path loss between the MTD and itself $\left(L_{m, u}\right)$ by comparing the received and the transmission power. Only the UEs having $L_{m, u}<L_{t h}$ participate in the contention process (these UEs are called relay candidates). Each relay candidate responds to the MTD with a RelayCandidate (RC) packet. To send its RC packet, the relay candidate chooses randomly and uniformly one timeslot $s \in[1, W]$ in the window. The first relay candidate that transmits an RC packet without collision wins the contention process.

3) The MTD broadcasts the feedback packet as soon as it successfully receives an RC packet. The feedback 
packet serves to confirm the winning UE and to avoid the hidden node problem. The hidden node problem occurs when a relay candidate is not able to hear the $\mathrm{RC}$ packet sent by another one. The relay candidate that wins the contention is called the selected relay while the other relay candidates stop contending when they receive the feedback packet.

If the MTD finds a relay, it establishes a D2D link with the relay to transmit its data. Otherwise, the MTD transmits directly to the BS using a cellular communication. The D2D relaying procedure is represented in Fig. 2.

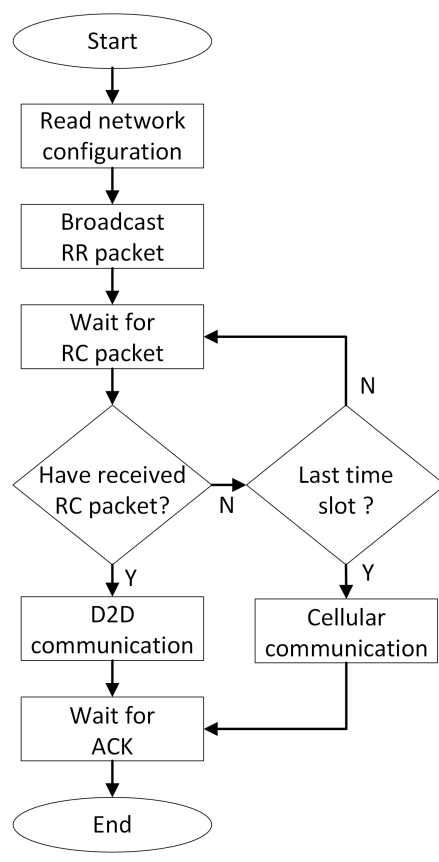

(a)

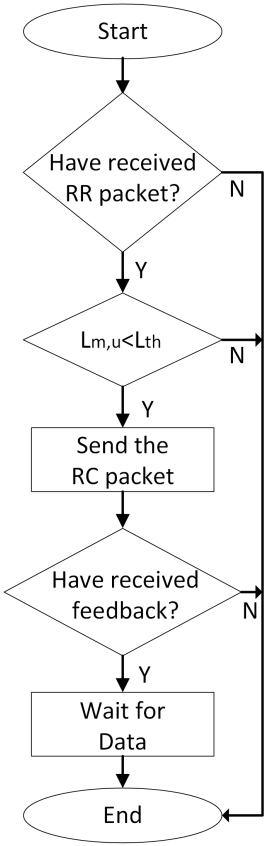

(b)
Fig. 2. D2D relaying procedure a) MTD side, and b) UE side.

We consider that the RR, RC, and feedback packets have a duration of one time-slot. We remark that there is no collision in the transmission of the RR packet since only the MTD transmits in that time slot. In other words, all UEs around the MTD receive the RR packet. However, when two or more UEs send the RC packet in the same time slot, we assume that the MTD is not able to decode these packets (ignoring the capture effect). Therefore, it has to listen during the next time slot.

The fact that the MTD sends the feedback packet and its data as soon as an RC packet is successfully received, on one hand, allows the MTD to save energy since the time in the active state is reduced. On the other hand, the relay candidates also save energy since, after sending the RC packet, if they do not receive the feedback packet they stop participating to the contention process as it means that there was a collision.

Fig. 3 shows the packet exchange sequence when the MTD selects a relay during the discovery phase. In this network, $W=6$ and there are four UEs that successfully received the RR packet. In slot 1 , no UE responds (No RC). In slot 2, there are two UEs responding at the same time (collision). In slot 3, only UE-4 transmits an RC packet (single slot) and thus wins the contention process. UE-1 and UE-3 stop participating in the process in slot 3 since they do not hear the feedback in this slot. UE-2, which had chosen slot 6, stops participating in the process when it hears the feedback packet in slot 4 .

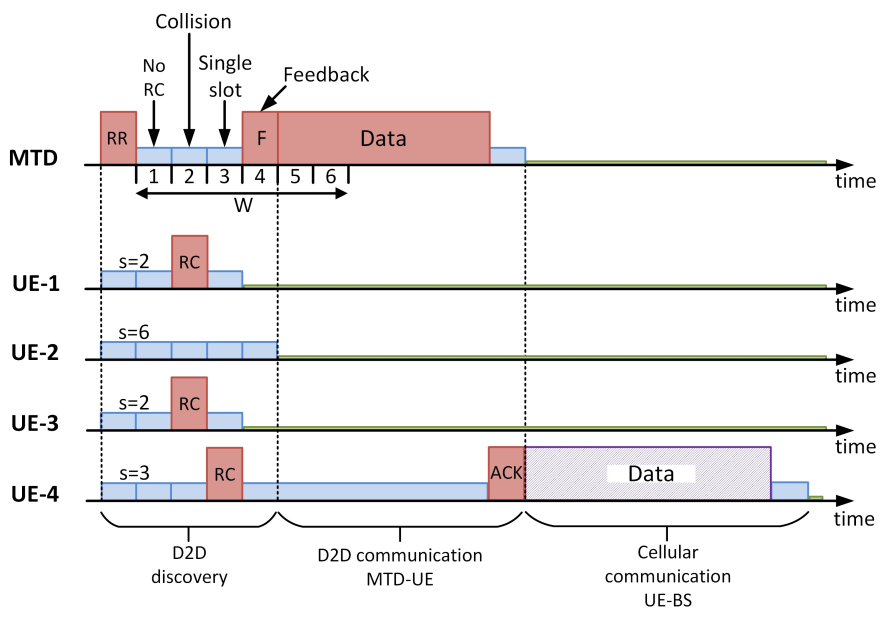

Fig. 3. Packet exchange sequence in the D2D relaying mechanism when the MTD selects a relay during the discovery phase.

\section{RELAY DISCOVERY PROBABILITY}

The relay discovery probability is the probability that the MTD finds a relay during the discovery phase.

Let $r$ be the distance between the MTD and a UE, the received power can be derived as $P_{r x}=P_{m} K_{d} r^{-\alpha_{d}}$, thus the MTD-UE path loss is $L_{m, u}=r^{\alpha_{d}} / K_{d}$, where $P_{m}$ is the MTD transmission power, $K_{d}$ and $\alpha_{d}$ are respectively the path loss factor and the path loss exponent for D2D communications. According to the discovery protocol, a UE that receives an $\mathrm{RR}$ packet participates in the contention process if and only if $L_{m, u} \leq L_{t h}$, where $L_{t h}$ is the path-loss threshold, which is a parameter sent by the MTD. Thus, a UE is a relay candidate when:

$$
r \leq\left(K_{d} L_{t h}\right)^{1 / \alpha_{d}} .
$$

We introduce a new parameter $R=\left(K_{d} L_{t h}\right)^{1 / \alpha_{d}}$ called radius of the discovery area. Thus, we define the discovery area (gray disk in Fig. 1) as the region bounded by a circle of radius $R$ centered on the MTD. All the UEs inside the discovery area are relay candidates.

The relay candidates choose a time slot $s \in[1, W]$, with a probability of $1 / W$ (uniform random choice). Since the locations of UEs form a homogeneous PPP with density $\lambda_{u}$, in each time slot $s$, the relay candidates form an independent thinning PPP with density $\lambda_{u} / W$. Thus, the probability of receiving $n \mathrm{RC}$ responses in a time slot is

$$
\mathbb{P}(N=n)=\frac{\left(\pi R^{2} \lambda_{u} / W\right)^{n}}{n !} \exp \left(-\pi R^{2} \lambda_{u} / W\right),
$$

A successful relay discovery occurs when at least one RC packet is successfully received (single slot) by the MTD. A 
single slot is a time slot where only one UE transmits an RC packet (i.e., a time slot without collision). Thus, the probability that a time slot $s$ is a single slot is

$$
P_{1}=\mathbb{P}(N=1)=\frac{N_{u}}{W} \exp \left(-\frac{N_{u}}{W}\right),
$$

where $N_{u}=\pi R^{2} \lambda_{u}$ represents the mean number of UEs inside the discovery area.

The relay discovery probability $P_{\text {disc }}$ is equivalent to the probability of finding at least one single slot during the contention process:

$$
P_{\text {disc }}=1-\left(1-P_{1}\right)^{W}=1-\left(1-\frac{N_{u}}{W} \exp \left(-\frac{N_{u}}{W}\right)\right)^{W}
$$

Taking the first derivative of $P_{\text {disc }}$ with respect to $N_{u}$, it is easy to show that the relay discovery probability is maximized when $W=N_{u}$. For $W=N_{u}, P_{1}=e^{-1}$ and $P_{\text {disc }}=1-$ $(0.63)^{W}$.

\section{NUMBER OF SLOTS USED IN THE CONTENTION PROCESS}

The maximum number of slots in the contention process is $W$. However, according to the discovery protocol, the contention process ends as soon as an RC packet is successfully received (i.e., at the first single slot). In case that none of the $\mathrm{RC}$ packets is correctly received, the number of slots used in the contention process is $W$.

Let $S$ be a discrete random variable with range $\{1,2, \ldots, W\}$ that represents the number of slots used in the contention process. This number of slots is equivalent to finding the first single slot. From (3), the probability that a time slot is a single slot is $P_{1}=\left(N_{u} / W\right) \exp \left(-N_{u} / W\right)$. Since $P_{1}$ is independent for each time slot, $S$ can be modeled as a geometric distribution with parameter $P_{1}$. Thus, the probability mass function (PMF) of $S$ is

$$
\mathbb{P}(S=s \mid W)= \begin{cases}\left(1-P_{1}\right)^{s-1} P_{1} & \text { if } 1 \leq s \leq W-1, \\ \left(1-P_{1}\right)^{W-1} & \text { if } s=W\end{cases}
$$

$\mathbb{P}(S=W \mid W)=\left(1-P_{1}\right)^{W-1}$ needs special attention because it is the sum of two probabilities, the probability that slot $W$ is a single slot $\left(1-P_{1}\right)^{W-1} P_{1}$ and the probability that none of the RC packets is correctly received $\left(1-P_{1}\right)^{W}$ by the MTD.

Let $\bar{S}$ be the mean number of slots used in the contention process. It can be easily calculated from the PMF of $S$ :

$$
\bar{S}=\sum_{s=1}^{W-1} s \mathbb{P}(S=s \mid W)+W \mathbb{P}(S=W \mid W)
$$

Substituting (5) into (6), and simplifying we obtain

$$
\bar{S}=\frac{1-\left(1-P_{1}\right)^{W}}{P_{1}},
$$

where $P_{1}=\left(N_{u} / W\right) \exp \left(-N_{u} / W\right)$.

The energy consumption in the discovery phase is directly proportional to $\bar{S}$. Therefore, we can minimize the energy consumption in the discovery phase by minimizing $\bar{S}$. Taking the first derivative of $\bar{S}$ with respect to $N_{u}$, we can find the minimum number of time slots in the contention process. Thus, solving $\frac{\partial \bar{S}}{\partial N_{u}}=0$, we deduce that the number of time slots in the discovery phase is minimal when $W=N_{u}$. And we obtain

$$
\min \bar{S}=e\left(1-\left(1-e^{-1}\right)^{W}\right) .
$$

Note that when $W$ is large $\min \bar{S} \approx e$.

\section{ENERGY CONSUMPTION IN CELLULAR AND D2D MODES}

An MTD can operate in one of the following states: Active (Tx or Rx), Idle, or Sleep. Each state has different and constant power requirements [13]. In our study, we analyze the energy consumed by the MTD when it transmits data (i.e., when it is in active state). Thus, the energy consumed by the MTD is computed as $E=P_{m, T} t_{m, T}+P_{m, R} t_{m, R}$, where $P_{m, T}$ and $P_{m, R}$ are the MTD power consumption (energy consumption per unit time) in Tx state and Rx state, respectively; $t_{m, T}$ and $t_{m, R}$ are the proportion of time spent in Tx state and Rx state, respectively. Note that $P_{m, T}$ is constant since we consider a fixed transmission power.

\section{A. Energy consumption in cellular mode}

In order to compute the energy consumed by the MTD when it transmits its data directly to the BS, we need to determine the transmission time (i.e., the time spent in Tx state) as in [4]. Assuming that the MTD transmits data message of total size $D$ bits, the transmission time is equal to $D / C_{m, b}$, where $C_{m, b}$ is the data rate (bits per second) in the MTD-BS link.

For computing the data rate $C_{m, b}$, we use a modified Shannon capacity formula proposed in [14]:

$$
C_{m, b}=B_{\text {eff }} B_{w} \log _{2}\left(1+\frac{\theta_{m, b}}{\theta_{\text {eff }}}\right),
$$

where $B_{w}$ is the transmission bandwidth, $B_{\text {eff }}$ adjusts for the system bandwidth efficiency of LTE, $\theta_{m, b}$ is the Signal-toNoise-Ratio (SNR) for the MTD-BS link, and $\theta_{\text {eff }}$ adjusts for the SNR implementation efficiency of LTE.

As we consider that the MTD transmits at fixed power $P_{m}$ the SNR expression for the MTD-BS link is then:

$$
\theta_{m, b}=\frac{P_{m} K_{c}\left(d_{m, b}\right)^{-\alpha_{c}}}{N_{0} B_{w}}
$$

where $N_{0}$ is the noise power spectral density, $d_{m, b}$ is the MTDBS distance, $K_{c}$ and $\alpha_{c}$ are respectively the path loss factor and the path loss exponent for cellular communications.

Thus, the MTD energy consumption due to the data transmission is

$$
E_{\text {cell }}=P_{m, T} \frac{D}{C_{m, b}},
$$

where $C_{m, b}=B_{\text {eff }} B_{w} \log _{2}\left(1+\frac{P_{m} K_{c}\left(d_{m, b}\right)^{-\alpha_{c}}}{N_{0} B_{w} \theta_{\text {eff }}}\right)$. 


\section{B. Total energy consumption in D2D mode}

The total MTD energy consumption in D2D mode is the sum of the energy consumed in the discovery phase and the energy consumed in the data transmission phase.

Since data rate adaptation requires the knowledge of channel state information (CSI), we consider that the discovery phase also serves to obtain the CSI of the MTD-UE link. Thus, we consider a fixed data rate during the discovery phase and a dynamic data rate adaptation during the data transmission phase.

1) Energy consumption in the discovery phase: We consider that the RR and feedback packets have a duration of one time-slot $\left(T_{s}\right)$. The MTD is in Tx state when it transmits the RR or the feedback packet, and it is in Rx state during the contention process. Thus, the average energy consumption during the discovery phase is

$$
\bar{E}_{\text {D2D,disc }}=T_{s}\left(2 P_{m, T}+\bar{S} P_{m, R}\right),
$$

where $P_{m, T}$ and $P_{m, R}$ are the MTD energy consumption in Tx state and Rx state, respectively; and $\bar{S}$ is the mean number of slot used in the contention process, which is defined in (7).

2) Energy consumption in the data transmission phase: Once the discovery phase has been completed, the MTD will transmit its data to the selected relay (D2D communication). In case no relay has been found, due to low relay density or collisions, the MTD will transmit directly to the BS as in traditional cellular communications. Thus, the average energy consumption during the data transmission phase can be derived as

$$
\bar{E}_{\text {data Tx }}=P_{\text {disc }} \bar{E}_{\mathrm{D} 2 \mathrm{D}, \mathrm{comm}}+\left(1-P_{\text {disc }}\right) \bar{E}_{\text {cell }},
$$

where $P_{\text {disc }}$ is the relay discovery probability, which is defined in (4), $\bar{E}_{\mathrm{D} 2 \mathrm{D}, \mathrm{comm}}$ is the average energy consumption in the D2D communication, and $\bar{E}_{\text {cell }}$ is the average energy consumption in cellular mode, which is defined in (11).

$\bar{E}_{\mathrm{D} 2 \mathrm{D}, \mathrm{comm}}$ depends on the MTD-Relay distance, which is a random variable since the locations of the UEs form a PPP. Let $X$ be a random variable that represents the distance between the MTD and the relay. Since we consider a uniform random choice of time slots in the contention process, all relay candidates have the same probability of being selected as a relay (without considering their locations). Thus, the cumulative distribution function $(\mathrm{CDF})$ of $X$ can be derived as

$$
F_{X}(x)=\mathbb{P}(X \leq x)=x^{2} / R^{2}, x \in[0, R],
$$

where $R$ is the radius of the discovery area. Thus, the probability density function (PDF) of $X$ is

$$
f_{X}(x)=2 x / R^{2}, x \in[0, R] .
$$

To compute the energy consumption in the D2D communication $\left(E_{\mathrm{D} 2 \mathrm{D} \text {,comm }}\right)$, we follow the same procedure used to derive (11). We consider that during the D2D communication the MTD transmits at fixed power $P_{m}$. Using the modified Shannon capacity formula [14], we obtain

$$
E_{\mathrm{D} 2 \mathrm{D}, \mathrm{comm}}=\frac{P_{m, T} D}{B_{\mathrm{eff}} B_{w} \log _{2}\left(1+\frac{P_{m} K_{d} x^{-\alpha} d}{N_{0} B_{w} \theta_{\mathrm{eff}}}\right)},
$$

where $x$ is the MTD-Relay distance, $K_{d}$ and $\alpha_{d}$ are respectively the path loss factor and the path loss exponent for D2D communications.

Thus, the average energy consumption in the D2D communication is computed as follows:

$$
\bar{E}_{\mathrm{D} 2 \mathrm{D}, \mathrm{comm}}=\int_{0}^{R} \frac{P_{m, T} D}{B_{\mathrm{eff}} B_{w} \log _{2}\left(1+\frac{P_{m} K_{d} x^{-\alpha_{d}}}{N_{0} B_{w} \theta_{\mathrm{eff}}}\right)} f_{X}(x) d x,
$$

where $f_{X}(x)=2 x / R^{2}$ is the PDF of $X(15)$.

Taking into account that the MTD transmits to its maximum allowed transmission power (i.e., $P_{m}=23 \mathrm{dBm}$ ) and the MTD-UE distance $x$ is short, we can assume:

$$
\log _{2}\left(1+\frac{P_{m} K_{d} x^{-\alpha_{d}}}{N_{0} B_{w} \theta_{\text {eff }}}\right) \approx \log _{2}\left(\frac{P_{m} K_{d} x^{-\alpha_{d}}}{N_{0} B_{w} \theta_{\text {eff }}}\right) .
$$

Then in (17) we have

$$
\bar{E}_{\mathrm{D} 2 \mathrm{D}, \mathrm{comm}}=\frac{P_{m, T} D}{B_{\mathrm{eff}} B_{w} R^{2}} \int_{0}^{R} \frac{2 x}{\log _{2}\left(\frac{P_{m} K_{d} x^{-\alpha_{d}}}{N_{0} B_{w} \theta_{\mathrm{eff}}}\right)} d x,
$$

Integral (19) is simplified as follows:

$$
\bar{E}_{\mathrm{D} 2 \mathrm{D}, \mathrm{comm}}=A e^{B} E_{1}(B),
$$

where $A=\frac{\ln (4) P_{m, T} D}{\alpha_{d} B_{\text {eff }} B_{w}}, B=\frac{2}{\alpha_{d}} \ln \left(\frac{P_{m} K_{d} R^{-\alpha_{d}}}{N_{0} B_{w} \theta_{\text {eff }}}\right)$, and $E_{1}(z)=\int_{z}^{\infty} e^{-t} t^{-1} d t$ is the exponential integral.

Then, substituting (4) and (20) in (13) we get

$$
\bar{E}_{\text {data Tx }}=\left(1-\left(1-P_{1}\right)^{W}\right) A e^{B} E_{1}(B)+\left(1-P_{1}\right)^{W} \bar{E}_{\text {cell }},
$$

where $P_{1}=\frac{\pi R^{2} \lambda_{u}}{W} \exp \left(-\frac{\pi R^{2} \lambda_{u}}{W}\right)$, and $\bar{E}_{\text {cell }}$ is defined in (11).

3) Total MTD energy consumption in D2D mode: The average of the total MTD energy consumption in D2D mode is

$$
\bar{E}_{\mathrm{D} 2 \mathrm{D}, \mathrm{total}}=\bar{E}_{\mathrm{D} 2 \mathrm{D}, \mathrm{disc}}+\bar{E}_{\text {dataTx }} .
$$

From (12) and (21), we observe that $\bar{E}_{\mathrm{D} 2 \mathrm{D} \text {,total }}$ depends on three variables: $d_{m, b}, W$, and $R$.

4) Minimization of the MTD energy consumption: We propose a discovery mechanism that aims to minimize $\bar{E}_{\mathrm{D} 2 \mathrm{D}, \text { total }}$. As discussed in sections III and IV, $\bar{E}_{\mathrm{D} 2 \mathrm{D} \text {,disc }}$ is minimal when $W=N_{u}$ (since $\bar{S}$ is minimal). Moreover, when $W=N_{u}$ the maximum relay discovery probability $\left(P_{\text {disc }}\right)$ is reached, and consequently $\bar{E}_{\text {dataTx }}$ is minimized since in data transmission phase when the MTD uses a relay it consumes less energy than when it transmits directly to the BS. Therefore the optimal contention window size, minimizing the total MTD energy consumption is $W^{*}=N_{u}$ and $\bar{E}_{\mathrm{D} 2 \mathrm{D} \text {,total }}$ depends now only on two parameters $\left(R\right.$ and $\left.d_{m, b}\right)$. 
Looking for the optimal radius of the discovery area that minimizes the total MTD energy consumption in the D2D mode, let $R=\rho d_{m, b}$, where $0<\rho<1$. We then look for an optimal $\rho^{*}$ value that minimize $\bar{E}_{\mathrm{D} 2 \mathrm{D}, \text { total }}$ when the distance between the MTD and the BS is $d_{m, b}$. It is not possible to get this value analytically. We thus use numerical computation to find $\rho^{*}$.

Fig. 4 shows the energy consumption in D2D mode as a function of the distance MTD-BS and the parameter $\rho$. From this figure, we observe (in red) that for each distance MTDBS there exists an optimal $\rho^{*}$ that minimizes the total MTD energy consumption.

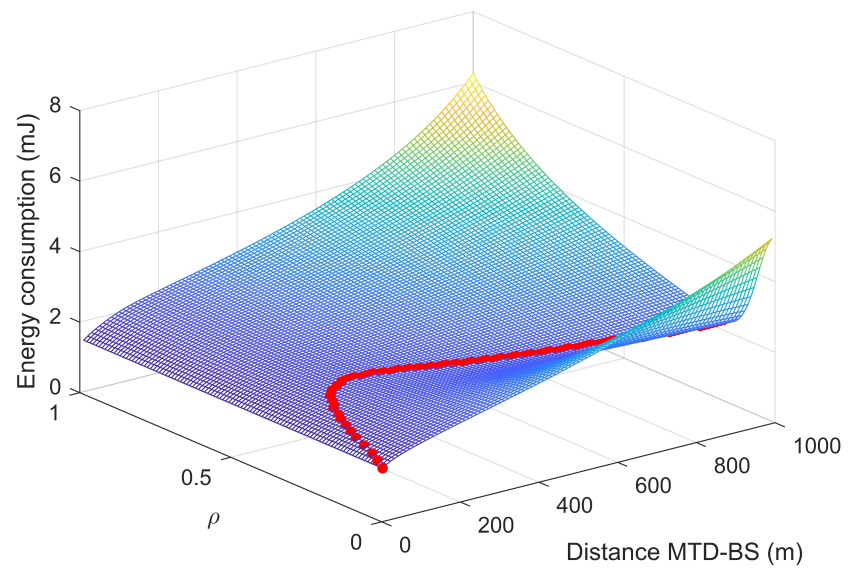

Fig. 4. Average total energy consumption in $\mathrm{D} 2 \mathrm{D}$ mode as a function of the distance MTD-BS $\left(d_{m, b}\right)$ and $\rho$, where $R=\rho d_{m, b}$ (radius of the discovery area), considering $D=200$ bytes and $\lambda_{u}=100 \times 10^{-6} \mathrm{UEs} / \mathrm{m}^{2}$.

We plot in Fig. 5 the optimal $\rho^{*}$ as a function of the distance MTD-BS for $\lambda_{u}=\left\{10 \times 10^{-6}, 100 \times 10^{-6}\right\} \mathrm{UEs} / \mathrm{m}^{2}$ and $D=200$ bytes.

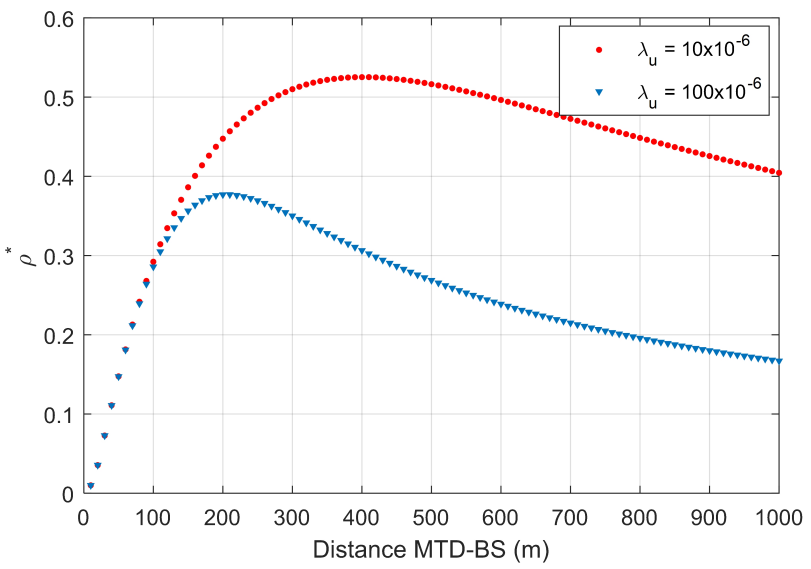

Fig. 5. Optimal $\rho^{*}$ as a function of the distance MTD-BS considering $D=$ 200 bytes and $\lambda_{u}=\left\{10 \times 10^{-6}, 100 \times 10^{-6}\right\} \mathrm{UEs} / \mathrm{m}^{2}$.

The optimal radius of the discovery area is

$$
R^{*}=\rho^{*} d_{m, b}
$$

TABLE II

SiMULATION PARAMETERS

\begin{tabular}{|c|c|}
\hline Parameter & Value \\
\hline MTD transmission power $\left(P_{m}\right)$ & $23 \mathrm{dBm}$ \\
\hline Discovery packet size $($ RR, RC, and feedback) & $12 \mathrm{bytes}$ \\
\hline MTD power consumption in Tx state $\left(P_{m, T}\right)$ & $925 \mathrm{~mW}$ \\
\hline MTD power consumption in Rx state $\left(P_{m, R}\right)$ & $200 \mathrm{~mW}$ \\
\hline MTD Bandwidth $\left(B_{w}\right)$ & $180 \mathrm{kHz}$ \\
\hline Noise Power Spectrum Density $\left(N_{0}\right)$ & $-174 \mathrm{dBm} / \mathrm{Hz}$ \\
\hline Carrier Frequency $\left(f_{c}\right)$ & $900 \mathrm{MHz}$ \\
\hline Cellular path-loss exponent $\left(\alpha_{c}\right)$ & 3.67 \\
\hline Cellular path-loss factor for a distance in meters $\left(K_{c}\right)$ & 0.0070 \\
\hline D2D path-loss exponent $\left(\alpha_{d}\right)$ & 4 \\
\hline D2D path-loss factor for a distance in meters $\left(K_{d}\right)$ & 0.0173 \\
\hline Time slot duration $\left(T_{s}\right)$ & $0.5 \mathrm{~ms}$ \\
\hline Bandwidth efficiency $\left(B_{\text {eff }}\right)$ & 0.56 \\
\hline SNR efficiency $\left(\theta_{\mathrm{eff}}\right)$ & 2 \\
\hline
\end{tabular}

From (3), we know that $N_{u}=\pi R^{2} \lambda_{u}$. Thus, the optimal contention window size is

$$
W^{*}=\pi\left(\rho^{*} d_{m, b}\right)^{2} \lambda_{u} .
$$

From (1), we can derive the path loss threshold $L_{t h}$ as follows:

$$
L_{t h}=\frac{\left(\rho^{*} d_{m, b}\right)^{\alpha_{d}}}{K_{d}} .
$$

Then, the two parameters that the MTD has to send in the RR packet are: $W^{*}$ and $L_{t h}$. The MTD-BS distance $\left(d_{m, b}\right)$ can be derived from the MTD-BS path-loss $\left(L_{m, b}\right)$ measured by the MTD.

\section{Numerical Results}

The analytical and simulation results are presented to validate the accuracy of our analytical models. The simulations are performed using MATLAB and the simulation parameters are specified in Table II. We use the MTD power consumption parameters proposed in [15], and we set the bandwidth efficiency $B_{\text {eff }}$ and the SNR efficiency $\theta_{\text {eff }}$ as the SISO channel parameters [14].

In Fig. 6 we show that the probability of finding a relay decreases as the mean number of UEs in the discovery area $\left(N_{u}\right)$ decreases. It is either due to a low UE density $\left(\lambda_{u}\right)$ or because the discovery area $\left(\pi R^{2}\right)$ is very small. The relay discovery probability increases as $N_{u}$ increases, until reaching a maximum. However, due to the limited number of time slots $(W)$, collisions also increase, reducing the relay discovery probability.

Fig. 7 shows the mean number of slots used in the contention process $\bar{S}$ as a function of $N_{u}$. We can see that the analytical and simulation results fit well. From this figure, we observe that when $N_{u}$ is zero (i.e., there is no UEs inside the discovery area), the number of time slots used in the contention process is $W$. This is because even if there is no relay candidate, the MTD waits for an RC packet until the last time-slot. We also note that as $N_{u}$ increases, $\bar{S}$ decreases and reaches a minimum value at $N_{u}=W$, and then $\bar{S}$ increases (i.e., a convex curve). This is because the probability 


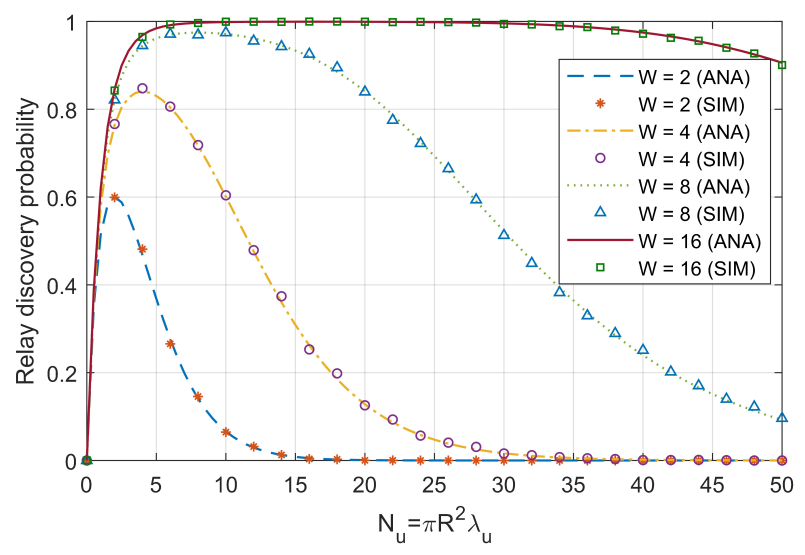

Fig. 6. Relay discovery probability as a function of the mean number of UEs inside the discovery area $\left(N_{u}\right)$ for $\mathrm{W}=\{2,4,8,16\}$.

of receiving an $\mathrm{RC}$ packet in the first slots increases as the number of relay candidates increases. But, if the number of relay candidates is greater than the number of slots available $(W)$, thus $\bar{S}$ increases due to the impact of the collisions.

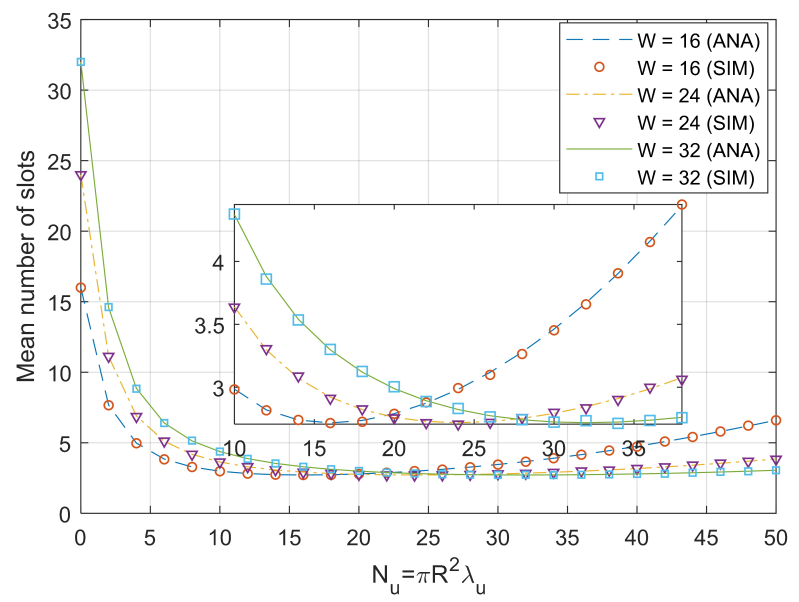

Fig. 7. Mean number of time slots used in the contention process $(\bar{S})$ versus the mean number of UEs inside the discovery area $\left(N_{u}\right)$ for $\mathrm{W}=\{16,24$, $32\}$.

In Fig. 8, we compare the energy consumption in cellular mode and the minimum average energy consumption in D2D mode (i.e., considering the the optimal values $W^{*}$ and $\rho^{*}$ ). This figure shows that the D2D relay mechanism allows reducing the energy consumption significantly when the data packet size is relatively large compared to the discovery packet size (12 bytes), and when the MTD is far from the BS.

When the data packet size is small (e.g. 50 bytes), the energy consumption in cellular mode is less than the energy consumption in D2D mode, because the energy consumed in the discovery phase is significant in comparison to the energy consumed in the cellular mode.

The energy reduction factor is defined as the ratio between the energy consumption in D2D mode and the energy con-

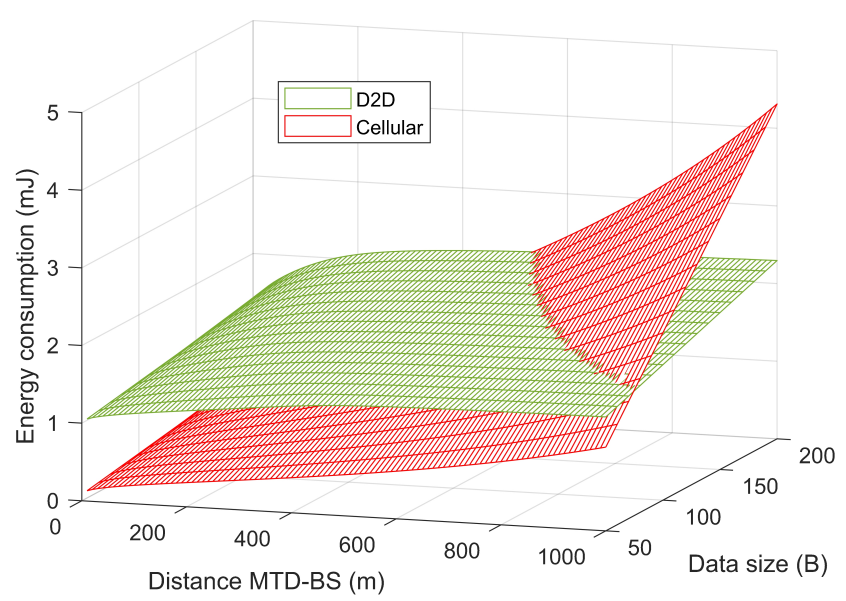

Fig. 8. Comparison of the total MTD energy consumption in cellular mode and in D2D mode as a function of the distance MTD-BS $\left(d_{m, b}\right)$ and the data size $(D)$, considering $\lambda_{u}=100 \times 10^{-6} \mathrm{UEs} / \mathrm{m}^{2}$.

TABLE III

ENERGY CONSUMPTION FOR CELLULAR AND D2D MODES

\begin{tabular}{|c|c|c|c|c|}
\hline Data size & \multicolumn{2}{|c|}{$\mathbf{1 0 0}$ bytes } & \multicolumn{2}{|c|}{ 200 bytes } \\
\hline Distance MTD-BS (m) & $\mathbf{5 0 0}$ & $\mathbf{1 0 0 0}$ & $\mathbf{5 0 0}$ & $\mathbf{1 0 0 0}$ \\
\hline \hline Energy consumption Cellular (mJ) & 1.06 & 2.16 & 2.12 & 4.31 \\
\hline Energy consumption D2D (mJ) & 1.65 & 2.68 & 2.11 & 2.16 \\
\hline Energy Reduction Factor & 1.56 & 0.78 & 1.00 & 0.50 \\
\hline
\end{tabular}

sumption in cellular mode. From Fig. 8, the energy reduction factor, for two different data sizes and for two different MTDBS distances, are obtained and shown in Table III. We observe that in D2D mode, the MTD consumes 50\% less energy than in cellular mode for $D=200$ bytes and $d_{m, b}=1000$ meters.

\section{CONCLUSION AND FUTURE WORKS}

In this paper, we analyze the total MTD energy consumption due to the transmission of data. We propose a simple distributed discovery protocol, which is efficient in terms of energy consumption. We show that using this D2D relay mechanism, the MTD energy consumption is significantly reduced when the data packet size is relatively large compared to the discovery packet size, and when the MTDs are far from the BS (unfavorable link budget). Furthermore, we derive analytical expressions for the relay discovery probability, the mean number of times slots used in the contention process, and the total MTD energy consumption. The accuracy of these analytical expressions is confirmed by simulations.

For future work, we want to extend our analysis of MTD energy consumption by taking into account the fading and shadowing effects. Moreover, we will investigate the MTD energy consumption using D2D relaying mechanisms considering the autonomous resource allocation mode for the discovery phase. 


\section{ACKNOWLEDGMENT}

This paper is part of and thereby supported by the Horizon 2020 project ONE5G (ICT-760809) receiving funds from the European Union. The authors would like to acknowledge the contributions of their colleagues in the project, although the views expressed in this contribution are those of the authors and do not necessarily represent the project.

\section{REFERENCES}

[1] 3GPP, "Study on new radio (NR) access technology physical layer aspects," TR 38.802, Mar. 2017.

[2] 3GPP, "Study on provision of low-cost Machine-Type Communications (MTC) User Equipments (UEs) based on LTE," 3rd Generation Partnership Project (3GPP), TR 36.888 V12.0.0, June 2003.

[3] "Proximity-Based Services (ProSe) (Release 14)," Third Generation Partnership Project (3GPP), Tech. Rep. TS 23.303, Dec. 2016.

[4] C. Vargas Anamuro, N. Varsier, J. Schwoerer and X. Lagrange, "Simple modeling of energy consumption for D2D relay mechanism," 2018 IEEE Wireless Communications and Networking Conference Workshops (WCNCW), Barcelona, 2018, pp. 231-236.

[5] G. Rigazzi, N. K. Pratas, P. Popovski, and R. Fantacci, "Aggregation and trunking of M2M traffic via D2D connections," in Communications (ICC), 2015 IEEE International Conference on, 2015, pp. 2973-2978.

[6] R. Madan, N. B. Mehta, A. F. Molisch and J. Zhang, "Energy-Efficient Cooperative Relaying over Fading Channels with Simple Relay Selection," in IEEE Transactions on Wireless Communications, vol. 7, no. 8, pp. 3013-3025, August 2008.

[7] Z. Zhou, S. Zhou, J. Cui and S. Cui, "Energy-Efficient Cooperative Communication Based on Power Control and Selective Single-Relay in Wireless Sensor Networks," in IEEE Transactions on Wireless Communications, vol. 7, no. 8, pp. 3066-3078, August 2008

[8] Y. Li, C. Liao, Y. Wang and C. Wang, "Energy-Efficient Optimal Relay Selection in Cooperative Cellular Networks Based on Double Auction," in IEEE Transactions on Wireless Communications, vol. 14, no. 8, pp. 4093-4104, Aug. 2015.

[9] X. He and F. Y. Li, "Cooperative RTS/CTS MAC with relay selection in distributed wireless networks," 2009 International Conference on Ultra Modern Telecommunications and Workshops, St. Petersburg, 2009, pp. $1-8$.

[10] A. Awang, X. Lagrange and D. Ros, "Toward an analysis of energy consumption in multihop wireless sensor networks," 2010 International Conference on Intelligent and Advanced Systems, Manila, 2010, pp. 1-6.

[11] Hongnian Xing and S. Hakola, "The investigation of power control schemes for a device-to-device communication integrated into OFDMA cellular system," 21st Annual IEEE International Symposium on Personal, Indoor and Mobile Radio Communications, Instanbul, 2010, pp. 1775-1780.

[12] ITU-R, "Guidelines for evaluation of radio interface technologies for IMT-Advanced," International Telecommunication Union, Tech. Rep. 2135-1, 2009.

[13] "Cellular system support for ultra low complexity and low throughput internet of things," Tech. Rep. 45.820 v13.1.0, 3GPP TSG GERAN, 2015.

[14] P. Mogensen, W. Na, I. Z. Kovács, F. Frederiksen, A. Pokhariyal, K. I. Pedersen, T. Kolding, K. Hugl, and M. Kuusela, "LTE capacity compared to the shannon bound," in Vehicular Technology Conference, 2007. VTC2007-Spring. IEEE 65th, pp. 1234-1238, IEEE, 2007.

[15] ONE5G project, deliverable D3.2, "Recommended Multi-Service Performance Optimization Solutions for Improved E2E Performance", to be published in May 2019. 